\title{
SPATIALLY BIASED EYE MOVEMENTS IN OLDER DRIVERS WITH GLAUCOMA AND VISUAL FIELD DEFECTS
}

\author{
David E. Anderson ${ }^{1}$, Deepta A. Ghate ${ }^{2}$, Sachin Kedar ${ }^{1,2}$, Matthew Rizzo ${ }^{1}$ \\ ${ }^{1}$ Department of Neurological Sciences; ${ }^{2}$ Truhlsen Eye Institute \\ University of Nebraska Medical Center, Omaha, NE, USA \\ Email: david.anderson@unmc.edu
}

\begin{abstract}
Summary: Patients with glaucoma are at greater driving safety risk due to visual field defects. These driving safety risks may be mitigated by compensatory eye movements. We measured spatial allocation of eye movements in a panoramic driving simulator in 8 drivers with glaucoma and 5 with suspected glaucoma. All completed a driving simulator visual field task under three separate conditions: (1) parked with a naturalistic background (Baseline condition); (2) driving on a rural highway (Driving condition); and (3) driving and completing a competing auditory attention task (PASAT condition). Results showed that: (1) drivers with larger binocular visual field defects showed more restricted, spatially biased eye movements, and (2) greater task load led to more spatially biased eye movements in drivers with larger binocular visual field defects. Findings provide preliminary evidence of eye movement patterns that may reflect compensatory behaviors in drivers with glaucomatous visual fields. Better understanding of the relationship between visual field deficits, eye movement patterns, and driving in glaucoma can help inform countermeasures to improve safety and mobility in drivers with visual impairments.
\end{abstract}

\section{INTRODUCTION}

Glaucoma is a potentially blinding optic neuropathy characterized by peripheral visual field loss. Drivers with glaucomatous visual fields are at a higher risk for poor driving performance and safety (Kwon et al., 2016; McGwin et al., 2005, 2015). Visual field loss severity is a strong predictor of poor driving performance (Bowers, Peli, Elgin, McGwin, \& Owsley, 2005; Kwon et al., 2016). Perception studies show glaucoma patients may be unaware of how their visual field deficits contribute to their inability to detect peripheral hazards (Hoste, 2003). Supporting these studies, glaucoma drivers commonly fail to detect traffic hazards in their peripheral vision (Crabb et al., 2010; Haymes, LeBlanc, Nicolela, Chiasson, \& Chauhan, 2008; Lee, Black, \& Wood, 2017). Thus, improving object detection in regions of glaucomatous visual field loss may serve as a critical benchmark for improving driving safety in glaucoma drivers.

Patients may develop compensatory behaviors to overcome perceptual deficits imposed by glaucomatous visual fields (Kasneci et al., 2014; Kübler et al., 2015). For example, glaucoma patients may generate more exploratory eye movement patterns or spatially bias eye movements towards regions of visual field defects. This hypothesis has received limited support in the context of driving (Kübler et al., 2015; Lee, Black, \& Wood, 2018; but see: Vega, Leeuwen, Vélez, Lemij, \& Winter, 2013). These studies demonstrated more exploratory eye movement patterns, such as increased saccade frequency and distance. Whether drivers with glaucomatous visual fields exhibit spatially biased eye movements remains unknown. 
Our goal was to begin evaluating evidence for compensatory eye movements in glaucoma drivers with binocular visual field defects. We used remote eye tracking technology to measure eye movement patterns while drivers completed a driving simulator visual field (DSVF) task (Anderson, Ghate, Kedar, \& Rizzo, 2017) under increasing driving task load. The DSVF uses a simple stimulus detection paradigm to probe regions of the visual field routinely tested by standard clinical perimetry. We sought to evaluate eye movements under low environmental information processing demands to rule out contributions from external factors on generated eye movement patterns. We hypothesized that drivers showing greater binocular visual field defects would show more spatially biased eye movements.

\section{METHODS}

Patients diagnosed with glaucoma or pre-glaucoma (suspects) were recruited from the Truhlsen Eye Institute at the University of Nebraska Medical Center (UNMC) in Omaha, NE. Glaucoma suspects (Prum et al., 2016) had normal visual fields; glaucoma patients had a range of visual field defects. All had corrected visual acuity of at least 20/40 in each eye and were experienced visual field takers with at least three stable (reliability indices better than $20 \%$ ) visual fields over the last 2 years. Subjects were excluded if they had a diagnosis of neurological disorder or cognitive impairment. This study was approved by the UNMC IRB.

Visual field testing was performed in the ophthalmology clinic using the 30-2 or the 24-2 SITA standard strategy in automated perimetry (Humphrey Field Analyzer II-I (HVF), Carl Zeiss Meditec Inc) as part of routine clinical care prior to recruitment for the study. HVF assessed the central 48 and 60 degrees visual field of view in the 24-2 and 30-2 strategies, respectively. The Best Location algorithm was used to integrate HVF monocular visual fields (I-HVF) into binocular visual fields (Nelson-Quigg, Cello, \& Johnson, 2000).

SENSEI (Simulator for Ergonomics, Neuroscience, Safety Engineering and Innovation), a DriveSafety (Salt Lake City, UT) RS-600 Research Simulator, provided the driving environment (Anderson et al., 2017). SENSEI is constructed around a full-sized automotive cab (2004 Ford Focus). SENSEI's cab is centered within 7 Ultra-HD (3840x2160) curved LED displays that provide a 290-degree panoramic field of view. Additional side and center rear-view LCD monitors provide a full 360-degree simulated environment. SENSEI is fully integrated with instrumentation for recording vehicle and driver performance measures.

\section{Driving Simulator Visual Field}

Methods for the DSVF have been reported elsewhere (Anderson et al., 2017). Briefly, the DSVF maps out grid locations spanning $60^{\circ}$ and $20^{\circ}$ of total horizontal and vertical visual angle, respectively. Grid locations were tested in random patterns, and each grid location was tested 4 times. Patients were instructed to press a red button on the steering wheel after detecting a stimulus. Hits were counted if the button was pressed prior to a subsequent stimulus presentation. Each DSVF was approximately 4 minutes, and each DSVF procedure was repeated twice.

Procedures. Driving scenarios were completed on a straight two-lane rural road, with a 3.6-meter lane width and $88.5 \mathrm{~km} / \mathrm{h}$ speed limit. All drives were completed on an empty road without any 
pedestrians, vehicles, or traffic lights. Drivers completed DSVF procedures under three different driving task load conditions: (1) Baseline - drivers performed DSVF procedures while parked; (2) Driving - drivers performed DSVF procedures while maintaining vehicle speed according to posted speed limits; (3) PASAT - drivers performed DSVF procedures while maintaining vehicle speed and concurrently performing the paced auditory serial addition task (PASAT), a demanding task that required sustained attention to a series of spoken numbers. Drivers were instructed to complete DSVF procedures without any fixation demands, allowing us to evaluate changes in eye movement patterns across driving task loads.

Analysis. Visual field index (VFI) estimates the unimpaired proportion of visual field, ranging between $0 \%$ (fully impaired) to $100 \%$ (fully intact). Response rates (0-4) and cortical magnification weights were used to calculate a global DS-VFI (Bengtsson \& Heijl, 2008) for constructed DSVF visual fields. Gray scales and DS-VFI estimates from the DSVF are comparable to those obtained in the clinic (Anderson et al., 2017).

\section{Eye Tracking}

Procedures. Eye position was recorded using a remote eye-tracking system (FOVIO; Eyetracking, Inc.) mounted above the dashboard. The eye tracker has visual accuracy of 0.5 degrees, and gaze angular range of -30 to 30 degrees horizontal and -15 to 30 degrees vertical. Calibration sequences were completed on a $3 \times 3$ grid pattern, spanning $30 \times 18$ degrees of visual angle on the center display. For calibration verification, drivers were instructed to fixate stimulus locations while the experimenter monitored a real-time feed of eye position overlaid onto the center channel display. Raw data were integrated into the simulator output files, resulting in a $60-\mathrm{Hz}$ sampling rate. Missing eye tracker data were marked as null values, and data quality was automatically generated as a coded value, where poor data quality was marked as a zero according to manufacturer standards.

Eye Tracker Simulator Mapping. We sought to map raw eye tracking coordinates to degrees of visual angle in the simulator. To this end, we designed a scenario to measure eye tracking coordinates at DSVF stimulus positions on the center display. Stimulus characteristics were identical to the DSVF scenario with the following exceptions: (1) only positions on the center display were tested; (2) each stimulus remained on the display until drivers pressed the left red button; (3) drivers were instructed to fixate each stimulus before responding; and (4) each stimulus position was tested twice. Data were collected and analyzed only during button presses. Median $\mathrm{x}$ - and $\mathrm{y}$-coordinates were obtained for each stimulus locations. For each equidistant contiguous stimulus location $(\mathrm{n}=38$ ), vector lengths were estimated from $\mathrm{x}$ - and $\mathrm{y}$-coordinate differences. The mean of all vector lengths represented the raw distance in eye tracking coordinates associated with 6 degrees of visual angle.

Preprocessing Eye Tracking Data. Missing or poor quality data (see Procedures above) were excluded from analysis. Changes in raw fixation position values were converted to degrees of visual angle based on the formula obtained from the Eye Tracker Mapping scenario. Individual fixations were counted when distance between eye positions exceeded 1 degree of visual angle and were longer than 100 milliseconds. Calculated summary statistics are reviewed in Table 1. 
Table 1. Summary statistics for eye movements

\begin{tabular}{|l|l|l|}
\hline Summary Statistic & Definition & Significance \\
\hline Fixation Duration & Mean duration of fixations in seconds & $\begin{array}{l}\text { Associated with processing time of information } \\
\text { content within fixation }\end{array}$ \\
\hline Saccade Frequency & Number of saccades per minute & $\begin{array}{l}\text { Frequently scanning environment for possible } \\
\text { hazards or safety critical information }\end{array}$ \\
\hline Saccade Distance & Mean distance (degrees) between each fixation & Maximizing scanning range within visual scene \\
\hline Horizontal Variance & Variance in x-coordinates of each fixation & $\begin{array}{l}\text { Distribution of left-to-right scanning patterns (e.g. } \\
\text { detecting traffic signs or pedestrians) }\end{array}$ \\
\hline Vertical Variance & Variance in y-coordinates of each fixation & $\begin{array}{l}\text { Distribution of up-to-down scanning patterns (e.g. } \\
\text { detecting stop lights or potholes) }\end{array}$ \\
\hline Saccade Angle Bias & $\begin{array}{l}\text { Circular correlation of saccade angles (1= high } \\
\text { spatial bias; 0= low spatial bias) (Figure 1) }\end{array}$ & $\begin{array}{l}\text { Bias of saccades toward region of space (e.g. either } \\
\text { always fixating to left and back to center or } \\
\text { distributing fixations across entire scene) }\end{array}$ \\
\hline
\end{tabular}

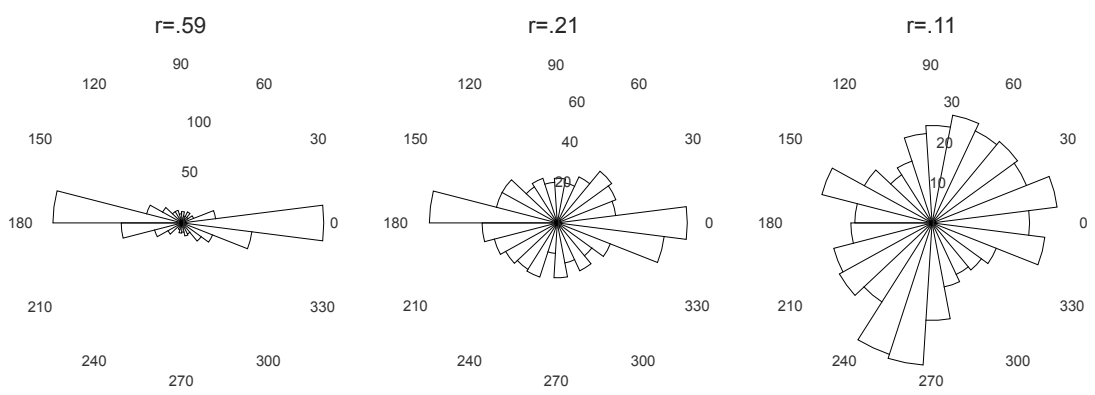

Figure 1. Examples of Saccade Angle Bias Calculations. Each polar plot contains a histogram of all saccade angles estimated for a given driver and DSVF condition. Saccade angle bias decreases from left-to-right, and the circular correlation estimate is provided above each plot.

\section{Statistical Analysis}

Analyses were performed with SAS Studio 3.6 (SAS Institute Inc.). Categorical data were descriptively summarized using frequency and percentage tables, and numeric data were descriptively summarized using means and standard deviations. Analyses were performed on eye movement summary statistics using generalized linear models (PROC MIXED). Repeated measures were modeled using a compound symmetry covariance structure. Kenward-Roger degrees of freedom corrections were used to account for missing data (Kenward \& Roger, 1997). Final models were derived using backward elimination stepwise regression, starting with a full factorial model of I-HVF, condition, and DS-VFI values. Post-hoc contrasts were assessed by comparing model-derived least squares means.

\section{RESULTS}

13 drivers (69\% women) completed driving simulation and eye tracking. Drivers were glaucoma patients with visual field defects $(n=8)$ or suspects without visual field defects $(n=5)$. Mean ages were $71.6 \pm 10.0$ (SD) and 52.2 \pm 16.6 , for glaucoma patients and suspects, respectively. HVF VFI estimates for glaucoma patients were $91.8 \pm 10.1$ and $72.8 \pm 22.1$ for best and worst eye, respectively. Welch-Satterthwaite t-tests revealed I-HVF estimates for glaucoma patients (87.6 \pm 8.7$)$ and suspects $(98.0 \pm 1.0)$ were statistically distinguishable $\left(\mathrm{t}_{7.3}=-3.36, \mathrm{p}=.0114\right)$, demonstrating binocular visual field loss in glaucoma patients. Less than $21 \%$ of raw eye 
tracking data were excluded due to missing or low quality data. Descriptive statistics for eye movement summary statistics are provided in Table 2.

Table 2. Descriptive statistics for eye movement summary statistics

\begin{tabular}{lllllll}
\hline & \multicolumn{3}{c}{ Glaucoma Patients } & \multicolumn{3}{c}{ Suspects } \\
& \multicolumn{1}{c}{ Baseline } & \multicolumn{1}{c}{ Driving } & \multicolumn{1}{c}{ PASAT } & Baseline & \multicolumn{1}{c}{ Driving } & PASAT \\
\hline Duration (seconds) & $0.48 \pm 0.48$ & $0.58 \pm 0.43$ & $0.62 \pm 0.80$ & $0.41 \pm 0.19$ & $0.67 \pm 0.27$ & $0.70 \pm 0.21$ \\
Frequency (\#/minute) & $53.9 \pm 29.8$ & $56.1 \pm 27.4$ & $47.1 \pm 24.6$ & $73.8 \pm 22.8$ & $50.5 \pm 26.1$ & $48.2 \pm 22.2$ \\
Distance (degrees) & $24.9 \pm 19.5$ & $21.9 \pm 16.5$ & $11.4 \pm 6.3$ & $28.1 \pm 12.3$ & $23.6 \pm 14.9$ & $14.6 \pm 11.6$ \\
Horizontal Variance & $1301 \pm 1445$ & $404.6 \pm 517.2$ & $159.7 \pm 157.9$ & $1462.9 \pm 629.7$ & $78.3 \pm 83.5$ & $63.6 \pm 41.9$ \\
Vertical Variance & $144.3 \pm 107.9$ & $684.0 \pm 590.6$ & $342.5 \pm 338.1$ & $259.4 \pm 185.9$ & $1103.2 \pm 880.5$ & $549.0 \pm 515.8$ \\
Saccade Angle Bias & $0.85 \pm 0.10$ & $0.69 \pm 0.28$ & $0.79 \pm 0.18$ & $0.87 \pm 0.05$ & $0.70 \pm 0.18$ & $0.60 \pm 0.15$
\end{tabular}

We observed an effect of $\operatorname{I-HVF}(\mathrm{F}(1,11.8)=11.25, \mathrm{p}=.0059)$ and condition $(\mathrm{F}(2,17.4)=4.50$, $\mathrm{p}=.027)$, as well as an interaction between $\mathrm{I}-\mathrm{HVF}$ and condition $(\mathrm{F}(2,17.4)=5.55, \mathrm{p}=.0136)$, on saccade angle bias. Post-hoc comparisons on condition revealed a significant reduction in saccade angle bias during Driving $(\mathrm{t}(16.8)=-3.32, \mathrm{p}=.0041)$ and PASAT $(\mathrm{t}(18.4)=-2.84, \mathrm{p}=.0108)$ conditions relative to the Baseline condition. When we decomposed the interaction, we found that the rate of decline in saccade angle bias as a function of I-HVF was greater during Driving $(\beta=-1.63 \pm 0.57 ; \mathrm{t}(17.4)=-2.85, \mathrm{p}=.0108)$ and PASAT $(\beta=-1.71 \pm 0.61 ; \mathrm{t}(18.2)=-2.80, \mathrm{p}=.0118)$ conditions relative to the Baseline condition.

We also observed an effect of condition on saccade distance $(F(2,19.6)=4.80, p=.0202)$ and horizontal variance $(\mathrm{F}(2,20.1)=10.14, \mathrm{p}=.0009)$. Post-hoc comparisons revealed: $(1)$ decreases in horizontal variance $(\mathrm{t}(18.9)=-3.65, \mathrm{p}=.0017)$ during Driving relative to Baseline conditions; (2) decreases in saccade distance $(\mathrm{t}(20.6)=-3.06, \mathrm{p}=.006)$ and horizontal variance $(\mathrm{t}(21.5)=-3.95$, $\mathrm{p}=.0007)$ during PASAT relative to Baseline conditions; and (3) decreases in saccade distance during PASAT relative to Driving conditions $(\mathrm{t}(19.6)=-2.21, \mathrm{p}=.0392)$. No effects were observed for fixation duration, saccade frequency, or vertical dispersion.

In summary, we found that drivers with larger binocular visual field defects showed greater saccade angle biases, which reflects a greater spatial bias in eye movements, and that increasing driving task load led to greater changes in saccade angle bias as a function of visual field defect.

\section{DISCUSSION}

We used remote eye tracking technology to measure eye movements while drivers completed a DSVF task under varying driving task loads. Supporting our hypothesis, we found that larger binocular visual field defects were associated with a greater spatial bias in eye movement patterns. Importantly, biases in eye movement patterns were not due to environmental information processing demands such as pedestrians, vehicles, or traffic lights and signs. These results provide preliminary evidence for compensatory eye movement patterns that may mitigate effects of visual field defects in glaucoma (Kasneci et al., 2014; Kübler et al., 2015). Further evidence on spatial biases in eye movements and how they relate to visual field defects, may improve understanding of driving behavior and safety in glaucoma.

One previous study of driving simulation in glaucoma evaluated eye movement patterns during a stimulus recognition task that presented single letters across 25 coordinates (Vega et al., 2013). 
These authors reported no difference in exploratory eye movement between glaucoma patients and healthy controls in an otherwise empty road. A second simulation study showed that glaucoma patients who passed a simulated driving test that included road hazards showed longer saccades, shorter fixation durations, and increased saccade frequency compared to those who failed (Kübler et al., 2015). These studies, in combination with the current results, suggest eye movement patterns are largely influenced by environmental information processing demands. Future studies from our lab will assess the relationship between spatially biased eye movements and simulated driving performance during road hazard scenarios in drivers with a range of visual field loss.

We found both patient groups showed a decrease in saccade distance and horizontal variance with increasing driving task load. These results are consistent with previous studies showing reductions in exploratory eye movement patterns with increasing cognitive load (Chapman \& Underwood, 1998; Recarte \& Nunes, 2000, 2003; Victor, Harbluk, \& Engströ, 2005). In addition, our measure of saccade angle biases demonstrated more spatially biased eye movements with increasing driving task load, suggesting cognitive load may also lead to more stereotyped eye movements during driving.

In conclusion, we provide preliminary evidence for spatially biased eye movement patterns in patients with glaucomatous visual field defects. Spatially biased eye movement patterns may reflect compensatory mechanisms proposed to overcome perceptual deficits. Further work is needed to determine whether spatial biases in eye movements: (1) predict regions of visual field loss; (2) overcome perceptual deficits; (3) change under high environmental information processing demands; and (4) can be explained by between-group differences in age.

\section{ACKNOWLEDGEMENTS}

We thank the subjects for their participation and patience. This study was supported by the Greater Plains IDeA-CTR grant NIGMS IU54GM115458-01, a Pilot Grant award from the Department of Neurological Sciences at UNMC, and by NIA R01 AG017177.

\section{REFERENCES}

Anderson, D. E., Ghate, D. A., Kedar, S., \& Rizzo, M. (2017). A New Method for Estimating Effects of Visual Field Loss in a Panoramic Driving Environment. Proceedings of the Ninth International Driving Symposium on Human Factors in Driver Assessment, Training, and Vehicle Design, 2-8.

Bengtsson, B., \& Heijl, A. (2008). A Visual Field Index for Calculation of Glaucoma Rate of Progression. American Journal of Ophthalmology, 145(2), 343-353.

Bowers, A., Peli, E., Elgin, J., McGwin, G., \& Owsley, C. (2005). On-road driving with moderate visual field loss. Optometry and Vision Science : Official Publication of the American Academy of Optometry, 82(8), 657-67.

Chapman, P. R., \& Underwood, G. (1998). Visual Search of Driving Situations: Danger and Experience. Perception, 27(8), 951-964. http://doi.org/10.1068/p270951

Crabb, D. P., Smith, N. D., Rauscher, F. G., Chisholm, C. M., Barbur, J. L., Edgar, D. F., \& Garway-Heath, D. F. (2010). Exploring Eye Movements in Patients with Glaucoma When 
Viewing a Driving Scene. PLoS ONE, 5(3), e9710.

Haymes, S. A., LeBlanc, R. P., Nicolela, M. T., Chiasson, L. A., \& Chauhan, B. C. (2008). Glaucoma and On-Road Driving Performance. Investigative Opthalmology \& Visual Science, 49(7), 3035.

Hoste, A. M. (2003). New insights into the subjective perception of visual field defects. Bulletin de La Societe Belge d'ophtalmologie, (287), 65-71.

Kasneci, E., Sippel, K., Aehling, K., Heister, M., Rosenstiel, W., Schiefer, U., \& Papageorgiou, E. (2014). Driving with Binocular Visual Field Loss? A Study on a Supervised On-Road Parcours with Simultaneous Eye and Head Tracking. PLoS ONE, 9(2), e87470.

Kenward, M. G., \& Roger, J. H. (1997). Small Sample Inference for Fixed Effects from Restricted Maximum Likelihood. Biometrics, 53(3), 983.

Kübler, T. C., Kasneci, E., Rosenstiel, W., Heister, M., Aehling, K., Nagel, K., ... Papageorgiou, E. (2015). Driving with Glaucoma. Optometry and Vision Science, 92(11), 1037-1046.

Kwon, M., Huisingh, C., Rhodes, L. A., McGwin, G., Wood, J. M., \& Owsley, C. (2016). Association between Glaucoma and At-fault Motor Vehicle Collision Involvement among Older Drivers. Ophthalmology, 123(1), 109-116.

Lee, S. S.-Y., Black, A. A., \& Wood, J. M. (2017). Effect of glaucoma on eye movement patterns and laboratory-based hazard detection ability. PLOS ONE, 12(6), e0178876.

Lee, S. S.-Y., Black, A. A., \& Wood, J. M. (2018). Scanning Behaviour and Daytime Driving Performance of Older Adults with Glaucoma. Journal of Glaucoma, 27(6), 1.

McGwin, G., Huisingh, C., Jain, S. G., Girkin, C. A., Owsley, C., \& Owsley, C. (2015). Binocular visual field impairment in glaucoma and at-fault motor vehicle collisions. Journal of Glaucoma, 24(2), 138-43.

McGwin, G., Xie, A., Mays, A., Joiner, W., DeCarlo, D. K., Hall, T. A., \& Owsley, C. (2005). Visual Field Defects and the Risk of Motor Vehicle Collisions among Patients with Glaucoma. Investigative Opthalmology \& Visual Science, 46(12), 4437.

Nelson-Quigg, J. M., Cello, K., \& Johnson, C. A. (2000). Predicting binocular visual field sensitivity from monocular visual field results. Investigative Ophthalmology \& Visual Science, 41(8), 2212-21.

Prum, B. E., Lim, M. C., Mansberger, S. L., Stein, J. D., Moroi, S. E., Gedde, S. J., ... Williams, R. D. (2016). Primary Open-Angle Glaucoma Suspect Preferred Practice Pattern( ${ }^{\circledR)}$ Guidelines. Ophthalmology, 123(1), P112-51. http://doi.org/10.1016/j.ophtha.2015.10.055

Recarte, M. A., \& Nunes, L. M. (2000). Effects of verbal and spatial-imagery tasks on eye fixations while driving. Journal of Experimental Psychology: Applied, 6(1), 31-43. http://doi.org/10.1037/1076-898X.6.1.31

Recarte, M. A., \& Nunes, L. M. (2003). Mental workload while driving: effects on visual search, discrimination, and decision making. Journal of Experimental Psychology. Applied, 9(2), 119-37.

Vega, R. P., Leeuwen, P. M. van, Vélez, E. R., Lemij, H. G., \& Winter, J. C. F. de. (2013). Obstacle Avoidance, Visual Detection Performance, and Eye-Scanning Behavior of Glaucoma Patients in a Driving Simulator: A Preliminary Study. PLOS ONE, 8(10), e77294.

Victor, T. W., Harbluk, J. L., \& Engströ, J. A. (2005). Sensitivity of eye-movement measures to in-vehicle task difficulty. Transportation Research Part F: Traffic Psychology and Behaviour, 8, 167-190. http://doi.org/10.1016/j.trf.2005.04.014 